\title{
Stimulation of autoimmunity by toll-like receptor ligands
}

\author{
A Limmer, R Ganss, N Garbi, B Arnold, G J Hämmerling
}

Ann Rheum Dis 2005;64:iv15-iv17. doi: 10.1136/ard.2005.042556

$\mathrm{H}$ ealthy individuals are known to harbour self-specific $\mathrm{T}$ cells against a variety of immunodominant selfpeptides-for example, myelin-basic protein (MBP) peptides. Yet, autoimmunity in a given individual is a rare event indicating that the self-reactive cells are somehow prevented from inflicting damage. ${ }^{1}$ Various experiments in which transgenic animals express both peripheral antigen and its cognate T cell receptor (TCR) have demonstrated that these self-antigens and their respective $\mathrm{T}$ cells are able to coexist without eliciting tissue damage. ${ }^{2-4}$ Similarly, in patients and mice with tumours that express specific antigens the tumours continue to grow in the presence of antigen specific T cells. ${ }^{5-8}$ These studies therefore suggest that the mere coexistence of an antigen and its cognate $\mathrm{T}$ cell is not in itself sufficient to produce an immune response which can subsequently eliminate the cells expressing the selfantigen. This coexistence raises several crucial questions-for example:

- What does it take to shift the balance from tolerant coexistence of autoreactive $\mathrm{T}$ cells and self-antigens to autoimmune destruction?

- Are the mechanisms underlying this process the same for autoimmunity and tumour destruction?

- Can we exploit this knowledge to devise specific immunotherapies against autoimmune disorders and tumours?

Anergy and regulatory $\mathrm{T}$ cells are known mechanisms capable of preventing activation of self-reactive T cells. In this report, we present evidence that additional mechanisms are operative within the innate immune system. In particular, toll-like receptor (TLR) activation is required for destruction of tissue by activated autoreactive T cells.

\section{A TWO STEP MODEL FOR ORGAN SPECIFIC AUTOIMMUNITY}

Several years back, we asked the simple question whether overcoming self-tolerance and activation of self-reactive cells would be sufficient to cause tissue damage. For this purpose, mice were generated expressing major histocompatibility complex (MHC) class $\mathrm{I} \mathrm{K}^{\mathrm{b}}$ molecules as a model antigen outside the thymus and exclusively on hepatocytes owing to the use of the liver specific albumin or C-reactive protein promoters. ${ }^{3}$ In addition, the mice were transgenic for a T cell receptor (Des.TCR) with specificity for the $\mathrm{K}^{\mathrm{b}}$ molecule. The rationale for using a class I molecule as an autoantigen is that MHC class I molecules present peptides from endogenously synthesised antigens, and that the MHC class I peptide complex is not supposed to travel to other cells in a form that can still be recognised by Des.TCR T cells. Thus, T cells have to interact directly with the cell expressing the MHC I molecule. These double transgenic mice harbour large numbers of $\mathrm{K}^{\mathrm{b}}$ reactive CD8 T cells, which are, however, tolerant as indicated by their failure to reject $\mathrm{a} \mathrm{K}^{\mathrm{b}}$ positive tumour or skin grafts. ${ }^{3}$ In this model, TCR unresponsiveness can be reverted to a responsive phenotype by simultaneous challenge with $\mathrm{K}^{\mathrm{b}}$ and interleukin (IL)-2, thus demonstrating breakage of tolerance towards a peripheral antigen in vivo. ${ }^{9}$ Mice in which tolerance was reversed were then able to reject $\mathrm{K}^{\mathrm{b}}$ positive grafts. Surprisingly, despite activation of self-reactive T cells and the rejection of $\mathrm{K}^{\mathrm{b}}$ positive grafts, there was no autoaggression against $\mathrm{K}^{\mathrm{b}}$ positive hepatocytes. Histological inspection revealed that $\mathrm{K}^{\mathrm{b}}$ reactive T cells failed to infiltrate the $\mathrm{K}^{\mathrm{b}}$ positive liver parenchyma. Thus, additional factors appear to be involved in extravasation and effector function of self-reactive lymphocytes.

Autoimmune diseases are frequently preceded by infections, which besides mimicking self-antigens may also cause inflammatory responses in the target organ. To investigate the importance of inflammatory responses as a cause of autoimmune pathology following breaking of tolerance, the double transgenic mice were treated with proinflammatory inducers such as Listeria, $\gamma$ irradiation, or TLR9 stimulation by cytosine-phosphorothioate-guanine-rich oligodeoxynucleotides (CpG-ODN)..$^{10}$ Indeed, now the autoreactive $\mathrm{T}$ cells massively infiltrated the liver parenchyma and caused tissue damage, as measured by release of alanine aminotransferase. We conclude that activated self-reactive T cells do not cross an unstimulated endothelial barrier in numbers sufficient for tissue damage. Instead, a proinflammatory environment "opens" the endothelial barrier, thus allowing $\mathrm{T}$ cell infiltration and tissue destruction. Thus, the two step model of organ specific autoimmunity comprises an initial activation of autoreactive T cells and the presence of proinflammatory signals in the target organ for increased extravasation of self-reactive $\mathrm{T}$ cells resulting in tissue damage. Such a scenario makes sense if one considers that the immune system wants to focus $\mathrm{T}$ cells at the site of an inflammatory response.

\section{REPEATED INFLAMMATORY RESPONSES ALONE CAN CAUSE AUTOAGGRESSION}

Liver dendritic cells, Kupffer cells, and liver sinusoidal endothelial cells among resident liver cells are known to express TLR9, explaining the strong effect of CpG-ODN on the liver microenvironment. The proinflammatory response in the liver was found to induce expression of costimulatory molecules, adhesion molecules, and to increase the amount of MHC molecules in hepatocytes, thus generating a phenotype similar to that of antigen presenting cells. Indeed, these activated hepatocytes were able to activate $\mathrm{K}^{\mathrm{b}}$ specific T cells, which then, in turn, attacked the hepatocytes and caused transient liver damage. ${ }^{10}$ Two important conclusions can be drawn from this observation. Firstly, $\mathrm{T}$ cell priming can take place outside lymphoid organs, provided that the microenvironment and stimulatory conditions are appropriate. Secondly, T cells activated by inflamed nonlymphoid tissue can cause autoaggression. Thus, an imbalance in the microenvironment of an organ may be sufficient to cause autoaggression (fig l). It should be noted, however,

Abbreviations: CpG-ODN, cyłosine-phosphorothioate-guanine-rich oligodeoxynucleotides; MHC, major histocompatibility complex; TCR, T cell receptor; TLR, toll-like receptor 


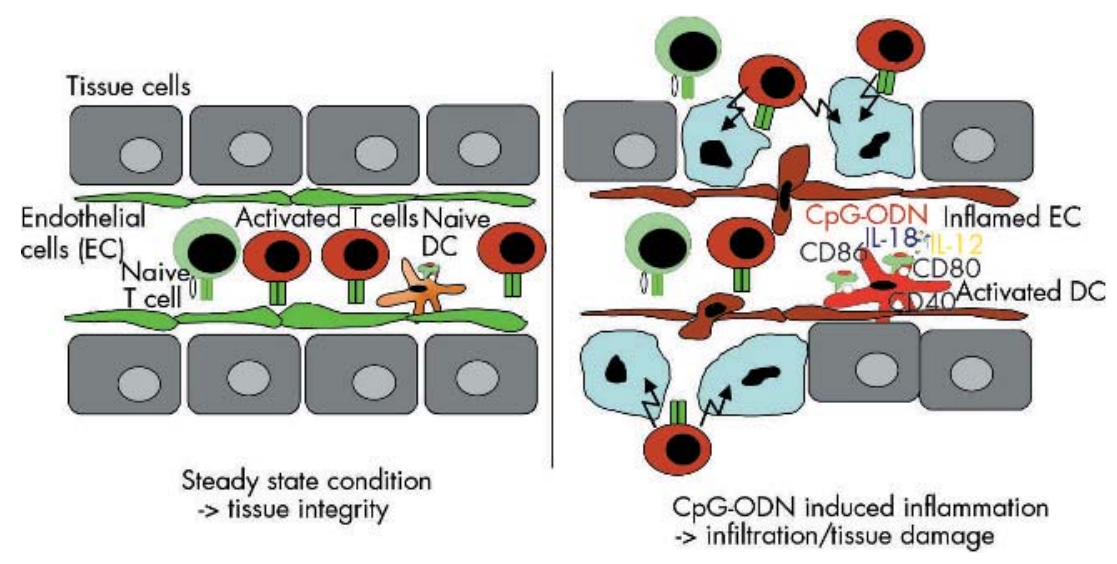

Figure 1 Inflammation may cause activation of T cells by tissue cells and autoaggression. Under steady state conditions circulating naive and activated T cells are prevented from entering tissues because of endothelial barriers (for example the liver parenchyma, but this scenario holds true for other organs and tumours). Although tissues may express autoantigens, tissue damage by autoreactive T cells is prevented. After an infection or contact of endothelial cells, macrophages, or dendritic cells (DCs) with toll-like receptor (TLR) ligands (for example CpG-ODN) in the relevant organ, inflammatory changes allow naive and activated T cells to enter tissues and destroy antigen bearing cells. Under inflammatory conditions it is also possible that naive T cells are activated directly in situ by non-professional tissue cells, which upregulate costimulatory molecules because of contact with inflammatory mediators produced by activated professional antigen presenting cells.

that the autoaggression observed here so far is only transient and does not result in serious autoimmune damage. Similar results supporting ours have recently been reported by Lang et $a l^{11}$ who show that TLR ligands conditioned the target organ via the induction of type I interferon, enabling activated $\mathrm{T}$ cells to become autoaggressive.

\section{TLR9 STIMULATION AND TUMOUR IMMUNITY}

Autoimmunity and tumour immunity can be regarded as comparable immune reactions, as they are both directed against a self-organ or self-organ-like tissue. In order to see whether the above mentioned two step model of autoimmunity (activation of self-reactive T cells plus a proinflammatory response) would also apply to tumour immunity, we used Rip.Tag tumour mice. These mice express the SV40 T antigen under the control of the rat insulin promoter (Rip) and develop insulinomas. The tumour nodules are characterised by a highly irregular vasculature and lack of cellular infiltrates. ${ }^{12}{ }^{13}$ Attempts to eradicate the tumours by vaccination or transfer of activated Tag specific $\mathrm{T}$ cells failed. Immunohistologic examination demonstrated that even the activated $\mathrm{T}$ cells did not transmigrate into the tumour tissue. To see if a proinflammatory stimulus would facilitate tumour rejection, we transferred tumour (Tag)-reactive CD4 and CD8 $\mathrm{T}$ cells into tumour bearing Rip.Tag mice in combination with $\gamma$ irradiation or CpG-ODN as proinflammatory stimuli. Indeed, after irradiation or treatment with CpG-ODN tumour endothelia were activated as indicated by intercellular adhesion molecule (ICAM) and vascular cell adhesion molecule (VCAM) upregulation, and massive T cell infiltration was observed..$^{14}$ As a consequence, the tumours were completely eradicated and the mice cured. Since tumour endothelia are not TLR9 positive, it was unclear how they were activated via CpG-ODN. Tumour resident macrophages were found to take up and respond to CpG-ODN, which apparently initiates a local proinflammatory response. ${ }^{15}$ These data suggest that in tumours the endothelial barrier prevents infiltration of activated $\mathrm{T}$ cells, similar to the observations made in the liver autoimmunity model described above. The findings may help to explain why in the clinic the success of immunotherapeutic treatment of tumours is still limited. Overcoming the endothelial tumour barrier may enhance immunotherapy of tumours. ${ }^{16}$

\section{CONCLUDING REMARKS}

The data presented here demonstrate that activation of selfreactive $T$ cells is not necessarily sufficient to cause autoimmunity-for example, autoimmune hepatitis. An additional step is required, namely activation of endothelia, which facilitates infiltration of autoreactivity cells into the liver parenchyma. Activation of endothelia can be achieved by the TLR9 ligand CpG-ODN. A similar mechanism is observed in tumour immunity. Tumour reactive $\mathrm{T}$ cells sometimes fail to infiltrate tumours. However, a proinflammatory stimulus such as CpG-ODN "opens" the endothelial tumour barrier, thereby permitting tumour eradication. Interestingly, induction of the local inflammatory milieu by CpG-ODN may modify the tissue in a way that otherwise non-professional cells acquire the ability to activate $\mathrm{T}$ cells outside lymphatic tissues. Similar modifications in tumours by TLR-L may help to stimulate $\mathrm{T}$ cell dependent tumour eradication.

\section{Authors' affiliations}

A Limmer, Institute for Molecular Medicine and Experimental Immunology, University of Bonn, Germany

R Ganss, N Garbi, B Arnold, G J Hämmerling, German Cancer Research Center (DKFZ), Tumor Immunology Program, Division of Molecular Immunology, Heidelberg, Germany

This work was supported by DFG (SFB405), EU (NoE MUGEN), and BMBF (NGFN2).

Competing interests: none declared

Correspondence to: G J Hämmerling, German Cancer Research Center (DKFZ), Heidelberg, Tumor Immunology Program, Division of Molecular Immunology, Germany; hammerling@dkfz.de

\section{REFERENCES}

1 Wucherpfennig KW, Zhang J, Witek C, Matsui, M, Mokabber Y, Ota K, Hafler DA. Clonal expansion and persistence of human T cells specific for an immunodominant myelin basic protein peptide. J Immunol 1994; 152:5581-92

2 Ohashi PS, Oehen S, Bureki K, Pircher H, Ohashi CT, Odermatt B, et al. Ablation of "tolerance" and induction of diabetes by virus infection in viral antigen transgenic mice. Cell 1991;65:305-17.

3 Schönrich G, Kalinke U, Momburg F, Malissen M, Schmitt-Verhulst A-M, Malissen B, et al. Down-regulation of T cell receptors on self-reactive $T$ cells as a novel mechanism for extrathymic tolerance induction. Cell $1991 ; 65: 293-304$. 
4 Lo D, Freedman J, Hesse S, Palmiter RD, Brinster RL, Sherman LA. Peripheral tolerance to an islet cell-specific hemagglutinin transgene affects both $\mathrm{CD} 4^{+}$ and $\mathrm{CD}^{+} \mathrm{T}$ cells. Eur J Immunol 1992;22:1013-22.

5 Hadden JW. The immunopharmacology of head and neck cancer: an update. Int J Immunopharmacol 1998;19:629-44.

6 Wick M, Dubey P, Koeppen H, Siegel CT, Fields PE, Chen L, et al. Antigenic cancer cells grow progressively in immune hosts without evidence for T cell exhaustion or systemic anergy. J Exp Med 1997;186:229-38.

7 Speiser DE, Miranda R, Zakarian A, Bachmann MF, Faienya-McKall K, Odermatt B, et al. Tumor antigens of peripheral solid tumors do not activate naive or primed T cells: implications for immunotherapy and autoimmune disease. J Exp Med 1997; 186:645-53.

8 Prévost-Blondel A, Zimmerann C, Stemmer C, Kulmburg P, Rosenthal FM, Pircher $\mathrm{H}$. Tumor-infiltrating lymphocytes exhibiting high ex vivo cytolytic activity fail to prevent murine melanoma tumor growth in vivo. J Immunol 1998; 161:2187-94.

9 Limmer A, Sacher T, Alferink J, Kretschmar M, Schönrich G, Nichterlein T, et al. Failure to induce organ-specific autoimmunity by breaking of tolerance: importance of the microenvironment. Eur J Immunol 1998;28:2395-406.
10 Sacher T, Knolle P, Nichterlein T, Arnold B, Hämmerling GJ, Limmer A. CpGODN-induced inflammation is sufficient to cause $T$ cell mediated autoaggression against hepatocytes. Eur J Immunol 2002;32:3628-37.

11 Lang KS, Recher M, Junt T, Navarini AA, Harris NL, Freigang S, et al. Toll-like receptor engagement converts $\mathrm{T}$-cell autoreactivity into overt autoimmune disease. Nat Med 2005;11:138-45.

12 Ganss R, Hanahan D. Tumor microenvironment can restrict the effectiveness of activated antitumor lymphocytes. Cancer Res 1998;58:4673-81.

13 Ryschich E, Schmidt J, Hämmerling GJ, Klar E, Ganss R. Transformation of the microvascular system during multistage tumorigenesis. Int J Cancer 2002;97:719-25

14 Ganss R, Ryschich R, Klar E, Arnold B, Hämmerling GJ. Combination of T cell therapy and trigger of inflammation induces remodeling of the vasculature and tumor eradication. Cancer Research 2002:62:1462-70.

15 Garbi N, Arnold B, Gordon S, Hämmerling GJ, Ganss R. CpG motifs as proinflammatory factors render autochthonous tumors permissive for infiltration and destruction. J Immunol 2004;172:5861-9.

16 Ganss R, Arnold B, Hämmerling GJ. Mini-review: Overcoming tumor-intrinsic resistance to immune effector function. Eur J Immunol 2004;34:2635-41. 